\title{
Structural insight into protein-aided bacterial biofilm formation
}

\author{
Yvette Roske $^{1}$, Anne Diehl ${ }^{2}$, Udo Heinemann ${ }^{1}$, Hartmut Oschkinat ${ }^{2}$, Kürsad Turgay ${ }^{3}$ \\ ${ }^{1}$ Max-Delbrueck-Center, Berlin, Germany, ${ }^{2}$ Research Institut for molecular pharmacology, Berlin, Germany, ${ }^{3}$ Institut for \\ Microbiologie, Leinitz University Hannover, Hannover, Germany \\ E-mail: yroske@mdc-berlin.de
}

Bacterial cells can form surface-associated sessile communities often described as biofilms. The development and establishment of this structured habitat is genetically programmed and consists commonly of exopoly $\neg$ Saccharides (EPS), extracellular DNA (eDNA), and proteins that form fibrils as major, stabilizing biofilm components. Additional biofilm proteins with specific functions such as hydrophobins were recently identified and characterized. Bacteria in biofilms are generally more resistant to environmental stress and less susceptible to antibiotics, hence infections associated with biofilm formation are more difficult to treat.

Biofilm-forming bacteria secrete proteins of very different primary structure that form protofibrils for supporting biofilm architecture. In combination with EPS, TasA is essential for the formation of a structured biofilm by Bacillus subtilis. TasA was first described as a spore-associated protein with antibacterial activity. Later it was identified as a major biofilm component whose extraction from the surface of $B$. subtilis yields oligomeric forms with an average molecular size of $600 \mathrm{kDa}$. Tas A preparations fibrillize on hydrophobic surfaces and at low $\mathrm{pH}$, suggesting that Tas $\mathrm{A}$ can form amyloid-like fibrils that stabilize the $B$. subtilis biofilm.

In Bacilli biofilm formation is genetically controlled by the regulatory repressor and anti-repressor proteins Sinl and SinR. In B. subtilis, the SinR controlled operon encoding the biofilm forming proteins contains the triade tapA-sipW-tasA. The peptidase SipW cleaves the signal peptides of those major biofilm-forming components prior to secretion of the proteins. Intriguingly, in pathogenic B. cereus or B. anthracis strains the tas A operon contains a SipW homologue together with Tas Alike genes that are closely related to those of the zinc-dependent metalloproteinase camelysin. The camelysins are secreted M73 peptidases (MEROPS), important for the pathogenicity of $B$. cereus and $B$. anthracis. Based on sequence alignments, TasA and camelysins can be considered homologues although TasA is not known for proteinase activity. Nevertheless, double deletion of both calY loci in $\mathrm{B}$. cereus results in a defect in biofilm formation.

In this work, we characterized the major biofilm protein of Bacillus subtilis, TasA in its mature secreted form and present the high-resolution structure of the folded domain. This model served as a template to model representatives of the homologous metalloprotease camelysin whose structure is not known. Based on the activity of structurally similar proteins, we investigated metal binding, proteolytic activity and oligosaccharide binding of TasA. However, we did not observe metal binding or significant protease activity of $B$. subtilis Tas A, which clearly distinguishes it from its homolog camelysin in $B$. cereus. Using a combination of biophysical analysis, NMR spectroscopy and X-ray crystallography, we identified and characterized three distinct structural states of the TasA protein. In particular, we have characterized oligomeric and fibrillar species observed under different conditions and compared it to the form observed in native biofilms.

Stover, A. G. \& Driks, A. (1999) J. Bacteriol 181, 1664-72. Secretion, localization, and antibacterial activity of TasA, a Bacillus subtilis spore-associated protein.

Kearns, D. B., Chu, F., Branda, S. S., Kolter, R. \& Losick, (2005) R. Mol Microbiol 55, 739-49. A master regulator for biofilm formation by Bacillus subtilis.

Mielich-Süss, B. \& Lopez, D. Environ. (2015) Microbiol. 17, 555-565. Molecular mechanisms involved in Bacillus subtilis biofilm formation.

Keywords: TasA, biofilm, structure 